\section{THE VALUE OF RADIOLOGY IN SOME INTRATHORACIC DISEASES.}

\section{By STANLEY MELVILLE,}

Director of the Radiological Departmenl, Brompton (Chest) Hospital ; Consulting Radiologist to King Edward VIIth Sanatorium, Midluurst; Consulting Radiologist to the Royal National Sanatorium, Ventnor; Honorary Radiologist to St. George's Hospital.

In a short article it will be the intention of the writer to suggest the value of this youngest of the ancillary aids to diagnosis in (a) Pulmonary tuberculosis, (b) pleuritic effusion ; $(c)$ neoplastic disease.

\section{Pulmonary Tuberculosis.}

The value of radiology in the diagnosis of tuberculous affections of the lung parenchyma would seem to have become an accepted fact. Most clinicians, and certainly all physicians with special knowledge of affections of the chest, appreciate the assistance that this branch of medical science gives them, and we have gone beyond the time when it was possible for great clinicians to doubt its diagnostic value in the early stage of pulmonary tuberculosis. The writer is inclined to think that any remaining suspicion of the value of radiography in this connection, rests largely with the man making the report, and it is up to the radiologist to study the question from every aspect with such care that he does not fall into the many pitfalls that await him and induce him to read into a skiagraph more than is well within the limits (very wide limits) of the physiologically normal ; not every adventitious shadow in the lung is pathological.

The present position of radiography in the diagnosis of pulmonary tuberculosis may be put something as follows: (I) Definite infiltration can be demonstrated upon an X-ray film at quite an early stage of the disease and frequently before definite physical signs are evident.

(2) X-ray evidence extending over a period of some months and consistently negative may be taken as conclusive evidence of the non-existence of pulmonary tuberculosis.

(3) That, in cases in which physical signs are present, it may be assumed that the initial stage has passed, and in such cases the X-ray picture will show, as a rule, much more extensive clisease than can be demonstrated by the physical examination. This is especially true in the presence of deepseated lesions and where there is emphysema, both of which conditions are a handicap to the clinician. Thus it comes about that radiology is of service, not, heaven forefend, as in the slightest degree taking the place of the clinician, but as an invaluable aid, possibly the greatest of all ancillary aids in diagnosis. It would seem right to suggest (though writing as a radiologist there is the risk of prejudice creeping in) that the best method is for the clinical and the radiological examination to be undertaken by separate individuals. For this thought there are several reasons. Firstly, whichever is done first, the mental balance must be influenced; in the second place, the patient gets the benefit of two individual examinations, and consequently, opinions; in the third place, the clinician who becomes his own radiologist must lose that fineness of outlook, that power of summing up all the evidence that has been brought together. Those who know the work of the Law Courts will appreciate the analogy of that most trying person, the judge who will insist upon acting the advocate and thus prejudging his case.

To sum up, it would appear that the value of radiology lies in the confirmation (or otherwise) of clinical suspicion; in determining the extent of disease prior to the induction of artificial pneumothorax; in noting the effect of the latter procedure.

It would be almost a truism nowadays to 
assert that where hæmoptysis is due to tuberculosis of the lungs, infiltration can be demonstrated on the X-ray film. There are, however, so many forms of hæmoptysis that are not of tuberculous origin, that it is not correct to take it for granted that the tubercle bacillus is the offending organism. Such things as bronchial varix, cardiac disease, the indefinite familial hremoptysis and vague purpuric tendencies, not to mention aneurysm, bronchiectasis and neoplastic disease, are all responsible for definite and striking lung hæmorrhage. The common, acute, onset of a new growth of the lung with a brisk hæmoptysis, is all too common a picture. Within the last year, the writer has seen two patients in whom the chief clinical evidence was recurrent hæmoptysis and both the patients had been sent to a sanatorium on suspicion of pulmonary tuberculosis. One patient had the further clinical evidence of alteration in the breath sounds at the left base, and after careful investigation the cause was found to be due to the presence of a blastomycetes. The other patient had loss of breath sounds to some extent at one base, and after the intratracheal injection of lipiodol a small growth was found to be blocking the main descending bronchus; subsequent bronchoscopy also demonstrated this, and the growth (a small fibroma) was successfully removed and the patient did well, the hæmoptysis ceasing at once.

Before concluding this part of the article, it may be fitting to deal shortly with two schools of thought that would appear to have arisen since the birth of radiography. One school, and that by far the largest, following the teaching of all the great clinicians and pathologists, regards the initial lesion of pulmonary tuberculosis as beginning in the lung parenchyma, such invasion being rapidly followed by reaction in the bronchial lymph glands. The other school, including amongst its teachers men of the highest reputation and learning, maintains that most, if not all tuberculous lesions begin in the lymphatic glands and spread thence into the lung parenchyma. From this school has arisen the term "hilum tuberculosis." The more and the longer is one's experience, the less can one subscribe to this teaching. In children, with the greater tendency to necrosis and caseation of the lymph nodes, there may be a direct spread, but even this is surely an inhalation spread? Again, it has very frequently been noticed that after the production of an artificial pneumothorax, subsequent infiltration, if it takes place in the more sound lung, is often of the more central type, but even here the further spread is by inhalation.

Another and more serious heresy, though possibly the same heresy under another name, is that of so-called peribronchial phthisis, of which Sir James Kingston Fowler wittily remarks, "peribronchial phthisis is common in America but unknown in France." This statement is, however, not quite fair to America, for Wesseler and Jaches are most condemnatory of this line of thought. They draw attention to the linear striation (upon which this disease is based), being caused just as much by bloodvessels and by lymphatic ducts as by the bronchial tree shadows. Once more the radiologist, backed by some clinicians, has allowed his vision to be obsessed by an exaggeration of the linear markings and has ascribed to them a pathological significance. Thus round a feeble structure, grew a new disease; fortunate, possibly, for some persons who during the Great War achieved their desire and escaped military service, but nevertheless, short-lived and not heard of to-day.

\section{Pleuritic Effusion.}

If one disease more than another has of late been in the minds of us all, it has been that affection leading to effusion in the pleural cavity. The ordinary type of pleural effusion, whether bacillary in origin, or the result of chill, giving the classical evidences to the clinician (Ellis's curve, loss of tactile 
and vocal fremitus, displacement of heart, loss of vocal resonance and breath sounds with possibly regophony and Skodaic resonance) are sufficiently definite without the aid of the radiologist. After parencentesis, it may be necessary to determine by $\mathrm{X}$-ray examination either the amount of residual fluid or the presence of the underlying cause, e.g., tuberculosis of the lung. Even here there are some possible clinical difficulties and possibility of error, especially in children, but with these the present article has not space to deal. It is in encysted effusions, and especially those along the line of the interlobar septum that the aid of the radiologist is sought. Such effusions may be situated anywhere in the pleural cavity and may be either serous or purulent. The most common cause of the infection is pneumococcal, more rarely streptococcal and the remainder usually tuberculous.

Wesseler and Jaches divide encapsulated effusions into two groups :-

(a) Superficial or parictal-apical, axillary, central or basal.

(b) Concealed. (I) Between the lung and the diaphragm. (2) Between the lung and the mediastinum. (3) Interlobar.

The infrapulmonary or diaphragmatic empyemata are naturally difficult of recognition clinically, especially when they are situated on the right side. The fluid is encapsulated by adhesion of the lung either to the diaphragm or to the chest wall. Where a diagnosis has to be made between infrapulmonary empyema and liver abscess or subphrenic abscess, the high-domed and sharply-defined diaphragm is frequently diagnostic of the latter. As a rule, in basal or infrapulmonary empyema the fluid collects posteriorly, that is to say, between the dome of the diaphragm and the lower limit of the pleural sac, the main pleural cavity being shut off by adhesion.

The respiratory movement in the more mesial part of the chest being limited, it is more readily cut off by adhesion, thus encapsulated effusions are least common in this situation. The diagnosis is all but impossible by ordinary clinical methods, but the sharply-defined opacity near the hilum of the lung, in company with clinical evidence pointing to suspicion of effusion, is most striking.

Possibly the most puzzling of the encysted effusions are those situated in the region of the axilla. The oval or spindle-shaped opacity, with sharply-defined inner wall, is readily recognized. The clinical evidence here again may be perplexing, for, on account of the frequent fixation of the lung by adhesion to the edge of the empyema cavity, the usual absolute dullness to percussion is wanting; again, and especially where the effusion is lying posteriorly, a loud, almost tympanitic note may be heard anteriorly. Finally, many of the apparently axillary effusions, seen in the postero-anterior plane, are found to be interlobar effusions when viewed in the lateral plane. In modern technique great importance is placed upon the value of the examination in the lateral plane; indeed, in almost all forms of encapsulated effusion, this view is most informatory and, unless absolutely impossible, should never be omitted. Even with a very ill patient, this is readily done without moving the patient. One final word on the subject of pleural effusions. It is of the greatest importance to distinguish between an effusion in the general pleural cavity (encysted or otherwise) and an interlobar empyema, in regard to the question of treatment. An exploring needle may, with impunity, be inserted in the first condition. In the second condition this is not the case, having regard to the risk of infecting the general cavity and producing a pyothorax.

NeOPLASMS OF THE LUNG.

Prior to the advent of radiology, there were but few publications and very few discussions on intrathoracic neoplasms. Such references as were made were chiefly from the standpoint of morbid anatomy. Nor can there be surprise at this attitude. In 
the first place, intrathoracic new growths were regarded as being of extremely rare occurrence, and, in the second place, the hopelessness of the prognosis was not conducive to any great enthusiasm.

New growths of the lung are usually divided into two groups, namely, those that are benign and those that are malignant. It is well, however, to note that only in the pathological sense can any such distinction be drawn, for the most benign new growth will, in time, deprive the patient of life by a process of pressure. It is well to stress this point, if for no other reason than that the clinician and the radiologist may be alive to the urgent need for early diagnosis. In another place the writer made the suggestion that on the first evidence of even occasional breathlessness, that could not be accounted for, the aid of the radiologist should be sought.

The benign neoplasms are for the most part extrapulmonary and cause pressure symptoms by pushing the lung in front of them and but seldom directly invading it, but in time displacing the heart and mediastinum.

Of the commoner benign new growths fibroma and the teratomata or congenital inclusion cysts are the most common. The fibromata grow usually from the posterior thoracic wall and are shown to be welldefined opacities pushing the surrounding lung in front of them but not directly invading it. Such tumours are non-irritant and but rarely produce any pleuritic irritation. The diagnosis is readily made by collapsing the lung, when they will be seen to be extrapulmonary. Operation for their removal is now fairly common, and, if the growth is not of large size, i.e., if the diagnosis has been macle early, the result of surgical interference is very good.

Teratomata or congenital inclusion cysts may occur anywhere. In the thorax, the most common are of the type of dermoid cysts, mainly epiblastic in origin and contain any of the constituents of skin (hair, sebaceous material and even teeth or fragments of bone). They grow from the remains of the thymus or from the third or fourth branchial clefts and are consequently anterior in position. In situation they thus differ definitely from the fibromata. The dermoid cyst is much more irritant, and adhesions between the cyst and the surrounding pleura are common, making it a much more difficult matter to separate them from the lung tissue, which they invade. The shape of the dermoid cyst is generally irregular and not so rounded as the fibromata. The diagnosis is readily made by examination in the true lateral plane, the anterior position of the tumour being almost pathognomonic.

Unfortunately, the early clinical diagnosis of intrathoracic new growth (whether benign or malignant) is indefinite and at times even misleading. The only evidence in one case seen by the writer was a brisk hæmorrhage, which did not assist in diagnosis. The longer a dermoid cyst is left in the thorax, the more difficult must operation for its removal become, and this is to be regretted, for if surgery is to have a fair chance, the earlier the diagnosis, the better the outlook.

Of the malignant neoplasms we are concerned with carcinoma and sarcoma.

Carcinomata are by far the most common, indeed it is a matter of doubt if there is any positive evidence of primary sarcoma of the lung itself.

It is still a vexed question whether or no there is an actual increase in lung cancer. Is the apparent increase due rather to the greater facilities for diagnosis, and the fact that in the pre-radiological days, many cases were ascribed to pulmonary tuberculosis, particularly of the fibroid type? Even if this be the case, surely the pathologist would have noticed changes inconsistent with such a diagnosis. Curiously enough the association between chronic pulmonary tuberculosis and cancer of the lung has been noted by the writer in two cases within the 
past month : in both, an old tubercular lesion with excavation, and associated with it a definite carcinoma growing from the mediastinum. Friedländer found squamouscelled carcinoma in the wall of an old tuberculous cavity. It would seem then there is a definite increase and the writer can only judge by his own experience that the last few years have revealed shadows in the lung which had not been met with during nearly twenty years of steady work. It is certainly true that colleagues of the writer working in other chest hospitals in London are finding similar increase for which they can account in no other way than that it is real and not due to other factors.

Almost without exception, carcinoma of the lung has an acute history to start with or acute bronchial irritation appears to be set up. Increasing dyspnœa and unproductive cough are frequently the main clinical signs. Not infrequently, the early acute symptoms appear to subside for a time and the patient seems to be better. A brisk hæmoptysis may be an early clinical symptom. At times the discrepancy between the intensity of the physical signs present and the apparent well-being of the patient is of marked significance to the clinician.

Primary cancer of the lung originates either $(a)$ from the epithelial lining of a large bronchus, most commonly a main bronchus close to the bifurcation of the trachea; (b) less commonly from a smaller bronchus deeply situated, producing the diffuse infiltrating growth in which there is rapid dissemination and destruction of lung tissue. The latter is in all probability identical with another form which has been described, namely, the "cavernous carcinoma," in which necrosis has taken place in the centre of a large mass.

Upper Lobe Carcinoma. - The growth originates from the bronchial mucosa of a large bronchus and causes eventually stenosis of the tube. What the radiologist sees is not so much the growth itself but the collapse of that part of the lung supplied by the large bronchus involved, that is to say, the results of the stenosis. Even with the whole of the upper lobe collapsed and appearing absolutely opaque the growth itself may still be quite small. The collapsed lobe is sharply demarcated by the lesser interlobar fissure. The heart is usually drawn over to the affected side partly on account of the shrinking of the collapsed lung and partly by the other lung. In the early stages, with the exception of a peculiar spasmodic and unproductive cough, there is but little clinical evidence.

Hilar Carcinoma. - Some of the tumours originating near the hilum, instead of causing stenosis, appear to traverse the bronchial wall and to invade the lung. In these cases, met often in lower lobe carcinoma, there is considerable enlargement of the bronchial glands. The growth is most irregular in form and outline, and heavy linear processes are seen extending into the lung tissue. It is in the diagnosis of these conditions that most of the radiological errors have been made. At times they may even be mistaken for interlobar emphysemata, and nothing but the closest co-operation between clinician and radiologist can avert error.

Basal Carcinoma.-Here the area of lung involved is seen to be irregularly opaque and collapsed. Owing to the fact that degenerative processes take place more rapidly than in upper lobe carcinoma, it will be appreciated that the differential diagnosis between this condition and bronchiectasis and basal abscess is ex. tremely difficult. In a case in the writer's recollection, the final diagnosis was made only at the autopsy, when, after much difficulty, a minute fragment at the extreme periphery of at large friable and brokendown mass was found, which, microscopically, was carcinoma.

The diaphragm in new growth of the lung is an interesting study. If the growth originates in a main bronchus before it 
enters the lung, the phrenic nerve may be involved, giving the characteristically high diaphragm with paradoxical movement. If, on the other hand, there is any considerable collapse of lung due to blocking of the bronchus, the diaphragm on that side will be raised and almost immobile.

There are two aids to diagnosis of some value :-

(I) The production of an artificial pneumothorax for diagnostic purposes is a simple and harmless expedient, and, although not of invariable value, at times the greater density of the involved lung compared with that of mere collapse is striking.

(2) The intratracheal injection of lipiodol. Of the value of this procedure the writer cannot speak too highly. In case of complete bronchial stenosis the sharply defined limit of the opaque fluid is strikingly shown. In conditions in which the bronchus is not completely blocked, the fluid is seen to pass through the partially constricted tube, an irregular outline, even a "rat-tail" appearance being shown.

This appearance is so unlike anything else, that the writer is convinced that in it, evidence of the greatest value is obtainable.

Sarcoma.-Primary sarcoma of lung is of extreme rarity. In 1903 Rolleston and Trevor published a case of this nature, and one or two other authentic causes appear to have been seen. Metastasis from some undiscovered cause undoubtedly accounts for some, and a metastasis from a small mediastinal primary sarcoma may well have been mistaken. In addition, there would appear to be no doubt but that some tumours labelled sarcoma are in reality specimens of smaller-celled carcinoma.

Endothelioma of Pleura.-Primary endothelioma is rare - but the characteristic feature is chiefly blood-stained effusion which shows the typical cells. On production of an artificial pneumothorax, irregular: opacities on the pleura may be seen or this condition may be viewed directly with the thoracoscope.
Metastatic Tumours of Lung.-As a rule it is not possible to distinguish with any certainty between the metastases of sarcoma or of carcinoma. Metastatic sarcoma, in the majority of cases, follows a primary focus on the bony structures, and grows with alarming rapidity. Metastatic carcinoma, on the other hand, is of much slower growth. The rounded "puff-ball" shape and the fact that they are usually multiple are common to both.

In direct extension to the lung or rather to the mediastinal glands following breast carcinoma, the pleura is as a rule rapidly involved through direct pleural spread. It is generally recognized that cancer cells may remain dormant in the bronchial glands for months or even years.

New Growths of the Mediastinum.-Hare, in an excellent analysis of 520 cases of mediastinal disease, gave the following table :-

$\begin{array}{llllr}\text { Cancer } & \ldots & \ldots & \ldots & \text { I } 34 \\ \text { Sarcoma } & \ldots & \ldots & \ldots & 90 \\ \text { Lymphadenoma } & \ldots & \ldots & \text { 2 I } \\ \text { Dermoid } & \ldots & \ldots & \ldots & \text { I } \\ \text { Hydatid } & \ldots & \ldots & \ldots & 8\end{array}$

The rest were composed of inflammatory diseases and included instances of lipoma, enchondroma and gumma.

The differential diagnosis is not (from an $\mathrm{X}$-ray point of view) without difficulty. Even to the clinician the physical signs are alike in most forms and such as there are, chiefly due to pressure effects.

Cyanosis, venous engorgement and signs of collateral circulation are possibly more common in neoplasm. The time factor is of service; if signs have persisted for eighteen months or thereabouts, the evidence is in favour of aneurysm.

The differential diagnosis has to be made between Hodgkin's disease, aneurysm of the aortic arch, sarcoma growing either from the mediastinal glands or possibly from the remains of the thymus or from the thyroid.

Lymphadenoma.- It is seldom that glandular enlargement in Hodgkin's disease is 
confined to the mediastinum; the associated soft and potato-like swellings met with elsewhere in the body are usually characteristic. A moderate eosinophilia and associated anæmia with occasional fever and increase in the leucocyte count are frequently in the clinical picture. The $\mathrm{X}$-ray evidence is that of a fairly well-defined mass on one or both sides of the mediastinum.

Aneurysm of the Aortic Arch.-Pulsation of the tumour was at one time regarded as being pathognomonic of this affection. It is, however, not infrequently a matter of considerable difficulty to decide whether a large, pulsating tumour in the mediastinum would turn out to be an aneurysm of the aorta or a new growth with transmitted pulsation. The history, a positive Wassermann and examination in the oblique diameter are of much greater value; the absence of "clubbing" being the deciding factor in a case of aneurysm.

Sarcoma of the Mediastinum.-Sarcoma sooner or later will be seen to invade both sides of the mediastinal space and will be seen to cause compression or deviation of the trachea with definite signs of compression and interference with the circulation.

Tumour of the Thyroid.-A substernal thyroid when viewed in the lateral plane will reveal itself as a tongue-like process growing downwards and forwards, and depressing the arch of the aorta. Such tumours are of very rare occurrence.

The characteristic feature of tumours of the thyroid, whether benign or malignant, is the definite and readily observable lateral compression of the trachea.

\section{SURGICAL RESURRECTIONS-I.}

IT must have fallen to the lot of every surgeon of experience to meet with cases which proceeded to that pitch of desperation that it seemed impossible to believe that recovery could take place, in which, nevertheless, the forces of nature aided by the surgical art have triumphed over: the depressing influences and led to recovery. In some instances life may temporarily have seemed extinct, but has revived against all expectation. The relation of some of these cases should be of general interest, both as to the particular events which may have led up to the crisis and the means taken to combat the profound vital depression.

The first two cases I propose to relate illustrate recovery after profound circulatory failure due to hæmorrhage, and may possibly show something new in the technique of treatment.

\section{Case I-SEVERE HæMORRHAGE FROM THE BOWEL IN DYSENTERIC TYPHLITIS.}

This case was one of the most remarkable recoveries that $I$ have ever seen and at the time astonished me. The patient was a private in the Middlesex Regiment, between 30 and $4^{\circ}$ years of age. $\mathrm{He}$ was serving in an eastern part of the theatre of the Great War during its last year. The time was mid-July and the weather was exceedingly hot. Admitted to hospital as a walking patient, he was regarded as a trivial case suffering from occasional attacks of diarrhoea. He was given an aperient the day following admission, and two days later complained of abdominal pain and developed a temperature of $\mathrm{IOI}^{\circ} \mathrm{F}$. When I saw him on that day a tender lump could be felt in the right lower abdomen, and in view of the history I had little hesitation in diagnosing appendicitis. That same day I 